\title{
Insights into Adherence among a Cohort of Adolescents Aged 12-20 Years in South Africa: Reported Barriers to Antiretroviral Treatment
}

\author{
Mhairi Maskew, ${ }^{1}$ Matthew P. Fox,${ }^{1,2,3}$ Denise Evans, ${ }^{1}$ Darshini Govindasamy, ${ }^{1,4}$ \\ Lise Jamieson, ${ }^{1}$ Given Malete, ${ }^{1}$ Constance Mongwenyana, ${ }^{1}$ and Karl Technau ${ }^{5}$ \\ ${ }^{1}$ Health Economics and Epidemiology Research Office, Department of Internal Medicine, School of Clinical Medicine, \\ Faculty of Health Sciences, University of the Witwatersrand, Johannesburg, South Africa \\ ${ }^{2}$ Department of Global Health, Boston University School of Public Health, Boston, MA, USA \\ ${ }^{3}$ Department of Epidemiology, Boston University School of Public Health, Boston, MA, USA \\ ${ }^{4}$ Health Systems Research Unit, South African Medical Research Council, Cape Town, South Africa \\ ${ }^{5}$ Empilweni Services and Research Unit, Department of Paediatrics and Child Health, University of the Witwatersrand, \\ Rahima Moosa Mother and Child Hospital, Johannesburg, South Africa \\ Correspondence should be addressed to Mhairi Maskew; mmaskew@heroza.org
}

Received 11 August 2016; Accepted 5 October 2016

Academic Editor: Belete A. Desimmie

Copyright (C) 2016 Mhairi Maskew et al. This is an open access article distributed under the Creative Commons Attribution License, which permits unrestricted use, distribution, and reproduction in any medium, provided the original work is properly cited.

\begin{abstract}
Adolescents experience disproportionately high rates of poor ART outcomes compared to adults despite prolonged use of antiretroviral therapy in Southern African treatment programs, presenting a significant challenge to national attempts to meet the UNAIDS 90-90-90 targets for 2020. This cohort study among adolescents aged 12-20 years accessing ART care at two urban public-sector clinics in Johannesburg between September and November 2013 aimed to identify factors potentially associated with poor attendance at clinic visits. Patients were followed up through routine medical records to identify missed visits (failing to attend clinic within 30 days of scheduled visit date) up to 2 years after enrolment. We enrolled 126 adolescents on ART for a median of 6.3 years (IQR: 2.7-8.4). A total of 47 (38\%) adolescents missed a scheduled visit within 24 months of enrolment. Older adolescents (18-20 years) were more likely to miss a visit compared to adolescents aged $12-14$ years (risk ratio $(\mathrm{RR})=1.72$; 95\% CI: 1.00-2.95). Those who were identified to have difficulty in taking medication $(\mathrm{RR}=1.57 ; 95 \% \mathrm{CI}: 1.13-2.18)$ as a barrier to care were more likely to miss a visit compared to adolescents who did not. Awareness of treatment fatigue, challenges to taking ART, and caregiver difficulties is important when considering interventions to improve treatment outcomes among adolescents.
\end{abstract}

\section{Introduction}

Despite the incredible success of the large-scale public-sector provision of antiretroviral therapy (ART) to HIV-infected people in South Africa, failure to retain these patients on long-term treatment threatens to undermine the massive gains made since 2004 and remains one of the most critical obstacles to achieving the UNAIDS 90-90-90 targets for 2020 which are intended to be a key milestone towards ending the HIV epidemic by 2030 [1]. Adolescents have been identified as vulnerable populations in need of prioritizing for adherence to treatment $[2,3]$. Globally, an estimated 2 million adolescents (10-19 years) are living with HIV/AIDS, with more than $80 \%$ of these from sub-Saharan Africa [4]. The escalating HIV-infected adolescent population, which is attributed both to a high incidence of horizontally acquired HIV and to long-term survival following perinatally acquired HIV infection [5, 6], means we can expect to see large impacts of this age group on national HIV treatment programs in the coming years. The recognition of the particularly high risk faced by adolescent girls and young women has seen initiatives such as PEPFAR's DREAMS launch pilot projects involving evidence-based interventions intended to break the cycles of HIV transmission in this group [7]. 
Adolescence is a stage of transition from childhood to adulthood, associated with specific challenges (including puberty) and vulnerability (such as early sexual debut, HIV, and STI acquisition). In addition, coping with the clinical and psychosocial impacts of HIV imposes a substantial burden on this vulnerable group [8-11]. HIV-infected adolescents encounter several known economic barriers to accessing HIV care such as cost of transport and distance to health facility [12-14] and are often caught between pediatric and adult services unable to address their special health needs which include disclosure of HIV status, adherence support, stigma and discrimination, sexual and reproductive health, mental health care, and legal and social support [5, 15-17].

Scale-up of ART in South Africa over the past ten years has been impressive with an estimated 3.4 million HIVinfected individuals on ART by $2015[18,19]$. However, ART coverage for children in the region has historically been considerably lower among children compared to adults $(22 \%$ versus 55\%, resp., in 2010) [20] though coverage among children has improved with USAID 2014 estimates indicating that nearly $50 \%$ of children $<15$ years of age living with HIV in South Africa are receiving ART [21]. Retention to ART is one of the universal goals of HIV treatment programs, yet there are several indications that adolescents are experiencing disproportionately poor ART outcomes compared to adults including higher rates of mortality, loss to follow-up, and lower rates of virologic suppression compared to other age categories [22-27], raising concern over the impact this group may have on national efforts to achieve the 90-90-90 targets.

Despite this, there are no national level estimates of adolescent retention in care or transfer across clinics and it remains unclear as to which adolescents are most likely to drop out of care in South Africa. Recent work using routine laboratory data to create a national HIV cohort has demonstrated the emergence of a youth treatment bulge, increasing numbers of adolescents aged 10 years and older accessing ART [28]. Given these figures and poor treatment outcomes experienced by this group, understanding the potential obstacles to adherence and retention in treatment programs among adolescents is urgently warranted. This study aimed to identify barriers and facilitators to remaining in and adhering to the continuum of ART care among HIV-infected adolescents in Johannesburg, South Africa. Identification of such factors could enable HIV care programs in this setting to target interventions most likely to reduce losses from ART care among adolescent populations.

\section{Materials and Methods}

2.1. Study Design and Sites. This prospective cohort study of HIV-infected adolescents accessing ART care was conducted at two public-sector antiretroviral treatment facilities in Johannesburg, South Africa. Both sites provide care and treatment according to the South African Department of Health National Antiretroviral Therapy Guidelines [12]. Under these guidelines, eligibility criteria for ART differed by age of the child. Children eligible for ART included: (1) all children $<1$ year of age; (2) children aged between 1 and 5 years with a WHO clinical stage 3 or 4 condition, a CD 4 count of $25 \%$ or less, or an absolute CD 4 count $<750$ cells $/ \mathrm{mL}$; and (3) children aged between 5 and 15 years with a WHO clinical stage 3 or 4 condition or CD $4<350$ cells $/ \mu \mathrm{L}$. Adolescents $>15$ years were eligible for treatment with (1) a CD4 count $<200$ irrespective of clinical stage, (2) a CD 4 count $<350$ cells $/ \mathrm{mm}^{3}$ (in the presence of TB or pregnancy), or (3) a WHO stage 4 condition or MDR/XDR TB irrespective of CD4 count.

At the time of the study, the Empilweni Clinic [29, 30], a pediatric HIV care and treatment clinic based at the Rahima Moosa Mother and Child Hospital (RMMCH), was caring for approximately 400 adolescents aged 12-20 who were receiving $(>80 \%)$ first-line antiretroviral treatment; the remainder of the group were receiving either second treatment or lamivudine-based holding regimens. Standard first-line regimens consisted of abacavir, lamivudine, and lopinavir/ritonavir for those $<3$ years of age or $10 \mathrm{~kg}$ in weight and abacavir, lamivudine, and efavirenz for those over 3 years of age and $10 \mathrm{~kg}$. The Themba Lethu Clinic (adult-based facility) is an outpatient antiretroviral treatment facility based at Helen Joseph Hospital, Johannesburg, Gauteng [31]. The site's patient population is primarily adult ( $99 \%$ aged $>20$ years) but at the time of the study ART and treatment monitoring were provided to approximately 80 adolescents. This includes a first-line regimen of tenofovir with lamivudine or emtricitabine plus either efavirenz or nevirapine.

2.2. Study Population. Eligible patients included HIVinfected adolescents aged between 12 and 20 years of age who were aware of their status and accessing ART treatment at either Empilweni Clinic or Themba Lethu Clinic regardless of duration of ART use. Identification of potentially eligible participants occurred during routine HIV care visits between September and November 2013 at specific clinic locations in which patients wait for relevant services (i.e., HIV counseling, doctor's visit, and pharmacy drug collection). Eligible and interested participants were provided with more detailed information about the study in a separate counseling room and individual consent (or participant assent plus parental or legal guardian consent for adolescents $<18$ years of age) was obtained. This recruitment strategy proceeded in a consecutive manner until the end of the enrolment period. All study participants received a small financial compensation in the form of a food voucher and parents or legal guardians who returned to complete a scheduled study appointment with an adolescent were also reimbursed for transport money.

2.3. Data Sources. Trained interviewers administered a structured questionnaire to each enrolled study participant. Though most questions were closed-ended, the questionnaire also included pairwise ranking and some open-ended questions. Participant data from the questionnaire was entered into a study database using CSPro software by trained data capturers. Adolescents were asked whether they considered several factors (related to caregivers, travel to clinic, psychosocial elements, the health care facility, or the treatment itself) as potential barriers to care during the interview. Routinely collected medical record data (including clinic visit dates, ART regimen history, and viral load results) were extracted from electronic medical records maintained by 
each of the study sites and linked to participant questionnaire data.

2.4. Study Variables and Analysis. The primary outcome was defined as missing a scheduled ART drug collection visit by more than 30 days. Several factors were investigated as potential predictors of poor adherence. These included the following: (1) demographic characteristics of the participant, (2) socioeconomic features of the participant's household, (3) caregiver issues, and (4) problems with service delivery at the treatment facility.

The characteristics of the study population were summarized with descriptive statistics including simple proportions for categorical variables and medians with interquartile ranges for continuous variables. The overall frequency of each of the factors reported during the interview as being considered by the adolescent as a potential barrier to care is presented. In addition, we present these frequencies stratified by treatment facility and, by proxy, model of care (pediatricbased compared to adult-based treatment sites). Next, associations between each of the potential predictors and missing a clinic visit are estimated with crude risk ratios and 95\% confidence intervals. Estimates were also adjusted for age, gender, mother as caregiver, and time on ART.

In secondary analysis, we defined prevalent unsuppressed viral load at the time of study enrolment as having a VL > 400 copies $/ \mathrm{mL}$ within 3 months prior to and 3 months after the interview date. A log-binomial regression model was fitted in order to determine risk factors associated with prevalent unsuppressed viral load.

Previous work has demonstrated heterogeneity within age substrata of adolescent populations in terms of treatment outcomes $[22,25]$. In order to evaluate differences in perceived barriers to care by stage of adolescence, the analysis of missed visits was further stratified by age category. Those aged between 12 and 17 years at the time of study enrolment were categorized as younger adolescents, while those aged 1820 years were categorized as older adolescents.

\section{Results}

In total, 206 adolescents were screened for potential enrolment into the study. Of these, a total of 126 adolescents (61\%) were enrolled in the study with a median of 6.3 years on ART (IQR 2.7-8.4): 19 adolescents from the adult-based facility (Themba Lethu clinic) and 107 from the pediatricbased facility (Empilweni Clinic). Of the remaining 80 not enrolled, $41(51 \%)$ did not meet eligibility criteria and 18 (23\%) were unable to consent, as their legal guardian was not present. Further $16(8 \%)$ refused to participate (5 were in a hurry, 4 were not interested, and 7 had no reason given) and the remaining 5 were not enrolled for unknown reasons. Similar overall proportions were enrolled at the pediatric-based $(27 \%)$ and adult-based $(24 \%)$ facilities. The demographic characteristics of the enrolled study participants are summarized in Table 1. Adolescents at the adultbased care facility were older (median: 18 versus 15 years) and predominantly female (68\% versus $54 \%$ ) and had received fewer years of ART (median: 1.2 versus 6.8 years) than those at the pediatric-based facility. Nearly three-quarters (73\%) were on first-line and the remaining quarter was on second-line regimens at the time of study enrolment.

3.1. Potential Barriers to Care. Overall, the factor most frequently reported during the interview as being considered a potential barrier to care was long travelling distance to the clinic (61\% agreed it was a potential barrier to care), followed by the possibility that the adolescent's attendance at clinic visits would be noticed by friends or members of the school (33\%), having an elderly caregiver (32\%), high transport cost of the trip to the clinic visit $(32 \%$,$) and long queues to wait in$ at the clinic (31\%) (Table 2).

Though the factors most frequently reported to be potential barriers to care were the same for both the adult-based site and pediatric-based site, the relative importance differed slightly; having an elderly caregiver was reported as a potential barrier to care as frequently as long travelling distances to the clinic among predominantly older adolescents at the adult-based site, while it was the least frequently reported of the five among those attending care at the pediatric facility. Very few of the interviewed adolescents agreed that being disinterested in care or not believing that ART helps would be a barrier to care or that unfriendly health care workers presented problems in accessing and remaining in care.

3.2. Adherence to Scheduled Visits. Adolescents completing the questionnaire were then followed up passively through routine medical record data for 24 months after study enrolment. Of the 126 adolescents enrolled, 2 did not return to their treatment clinic at all after the study interview. Among the 124 that did attend at least one clinic visit after study enrolment, 92 (74\%) remained in care through to the end of 24 months of follow-up, while 11 (9\%) had been lost from care and $21(17 \%)$ had transferred to another heath care facility. Overall, during the course of the 24 months of follow-up, $38 \%$ $(n=47)$ of the study participants missed a scheduled clinic visit by 7 days or more. The median time to first missed visit was 6.7 months (IQR 3.0-11.1) and $46.8 \%(n=22)$ of visits were missed within 6 months and further $34.0 \%(n=16)$ within a year after study enrolment.

Several demographic factors were associated with an increased risk of missing a clinical visit after study enrolment (Table 3). In crude analyses, among both of the older adolescent categories (15-17 and 18-20), the proportions missing a clinic visit were higher compared to the group of 12-14 years ( $41 \%$ and $49 \%$, resp., versus $28 \%$ ). Overall, the results suggest that older adolescents (15-20 years) were more likely to miss a visit compared to those in the group of 12-14 years (risk ratio $(\mathrm{RR})=1.59 ; 95 \%$ confidence interval $(\mathrm{CI}): 0.97-2.67)$ and adolescents in the group of 18-20 years were at highest risk of missing a clinic visit (RR: 1.72; 95\% CI: 1.00-2.95) though the estimates lacked precision and statistical significance. Adolescents with their mother as their primary caregiver were somewhat less likely to miss a visit (RR: $0.67 ; 95 \%$ CI: $0.43-1.06)$. When adolescents were asked if they agreed that certain factors could be barriers to care, those who agreed that potential barriers included having a caregiver with financial difficulty ( $47 \%$ versus $36 \%$ ), not having enough time for clinic 
TABLE 1: Demographics at study enrolment.

\begin{tabular}{|c|c|c|c|}
\hline & All $(N=126)$ & $\operatorname{TLC}(N=19)$ & $\mathrm{EC}(N=107)$ \\
\hline \multicolumn{4}{|l|}{ Gender; $n(\%)$} \\
\hline Male & $55(43.7)$ & $6(31.6)$ & $49(45.8)$ \\
\hline Female & $71(56.3)$ & $13(68.4)$ & $58(54.2)$ \\
\hline Age (years); median (IQR) & $15(13-18)$ & $18(18-19)$ & $15(13-17)$ \\
\hline \multicolumn{4}{|l|}{ Age group; $n(\%)$} \\
\hline $12-14$ & $54(42.9)$ & $2(10.5)$ & $52(48.6)$ \\
\hline $15-17$ & $34(27.0)$ & $2(10.5)$ & $32(29.9)$ \\
\hline $18-20$ & $38(30.2)$ & $15(78.9)$ & $23(21.5)$ \\
\hline \multicolumn{4}{|l|}{ Nationality; $n(\%)$} \\
\hline South African & $120(95.2)$ & $18(94.7)$ & $102(95.3)$ \\
\hline Foreign & $6(4.8)$ & $1(5.3)$ & $5(4.7)$ \\
\hline \multicolumn{4}{|l|}{ Highest school level; $n(\%)$} \\
\hline Secondary & $48(41.4)$ & $9(64.3)$ & $39(38.2)$ \\
\hline Primary & $68(58.6)$ & $5(35.7)$ & $63(61.8)$ \\
\hline \multicolumn{4}{|l|}{ Dwelling type; $n(\%)$} \\
\hline Informal & $17(13.5)$ & $0(0.0)$ & $17(15.9)$ \\
\hline Formal & $95(75.4)$ & $18(94.7)$ & $77(72.0)$ \\
\hline Care facility $^{*}$ & $14(11.1)$ & $1(5.3)$ & $13(12.1)$ \\
\hline Number in household; median (IQR) & $4(3-6)$ & $3(2-6)$ & $5(3-7)$ \\
\hline \multicolumn{4}{|l|}{ caregiver type; $n(\%)$} \\
\hline Mother & $66(52.4)$ & $11(57.9)$ & $55(51.4)$ \\
\hline Granny & $19(15.1)$ & $5(26.3)$ & $14(13.1)$ \\
\hline Aunt & $20(15.9)$ & $1(5.3)$ & $19(17.8)$ \\
\hline Children's home & $13(10.3)$ & $1(5.3)$ & $12(11.2)$ \\
\hline Other & $8(6.3)$ & $1(5.3)$ & $7(6.5)$ \\
\hline \multicolumn{4}{|l|}{ Caregiver employed; $n(\%)$} \\
\hline Yes & $79(62.7)$ & $13(68.4)$ & $66(61.7)$ \\
\hline No & $34(27.0)$ & $6(31.6)$ & $28(26.2)$ \\
\hline Time on ART (years); median (IQR) & $6.3(2.7-8.4)$ & $1.2(0.5-1.6)$ & $6.8(4.9-8.5)$ \\
\hline
\end{tabular}

${ }^{*}$ Care facility included children's home, hospice, or shelter.

visits (53\% versus 36\%), clinic visits being noticed by friends or the school ( $45 \%$ versus $35 \%$ ), and unfriendly health care workers ( $75 \%$ versus $37 \%$ ) more frequently missed a visit after study enrolment than those who did not identify these as barriers to care. Adolescents who agreed that having problems in taking the medication and becoming tired of taking the medication were barriers to care were also more likely to subsequently miss a visit $(\mathrm{RR}=1.77$; 95\% $\mathrm{CI}$ : $1.14-2.74$; and $\mathrm{RR}=1.77$; 95\% CI: 1.14-2.73, resp.). Estimates adjusted for age, gender, mother as caregiver, and time on ART were less precise but overall consistent with crude estimates (Table 3).

3.3. Prevalent Unsuppressed Viral Load. In total, 103 adolescents had a recorded viral load measurement within the defined prevalent virologic outcome window period (within 3 months prior to and 3 months after the interview date), and, of these, 32 (31\%) had a VL > 400 copies $/ \mathrm{mL}$. The frequency of prevalent unsuppressed viral load differed by several demographic factors (Table 4). A higher proportion of those in both older adolescent categories (38\% for $15-17$ years and 32\% for 18-20 years) experienced unsuppressed viral load at the time of study enrolment compared to those aged 12-14 years $(26 \%)$. Prevalent unsuppressed viral load also differed by gender (36\% males versus $27 \%$ females). Higher frequency of unsuppressed viral load was also noted among adolescents who agreed that certain factors were obstacles to accessing or remaining in care compared to those who did not agree. This included having an ill caregiver (50\% versus 30\%), expensive transport fees (39\% versus $28 \%$ ), and treatment fatigue $(41 \%$ versus 28\%). Provider-related factors including long queues and inconvenient clinic operating hours were not perceived as barriers among those who did not have a suppressed VL.

3.4. Stratification by Age Category. As noted earlier, older adolescents were more likely to miss a subsequent clinic visit after study enrolment than either of the other age categories 
TABLE 2: Frequency of reported factors, overall and by site.

\begin{tabular}{|c|c|c|c|}
\hline & All $(N=126)$ & Adult-based facility $(N=19)$ & Pediatric-based facility $(N=107)$ \\
\hline Median number of factors (IQR) & $4(3-6)$ & $4(3-8)$ & $4(3-6)$ \\
\hline Long distance to clinic; $n(\%)$ & $77(61.1)$ & $10(52.6)$ & $67(62.6)$ \\
\hline Visits noticed by friends/school; $n(\%)$ & $41(32.5)$ & $7(36.8)$ & $34(31.8)$ \\
\hline Caregiver is elderly; $n$ (\%) & $40(31.7)$ & $10(52.6)$ & $30(28.0)$ \\
\hline Transport fee is expensive; $n(\%)$ & $40(31.7)$ & $7(36.8)$ & $33(30.8)$ \\
\hline Long waiting queues at clinic; $n(\%)$ & $39(31.0)$ & $6(31.6)$ & $33(30.8)$ \\
\hline Visits noticed by family/community; $n$ (\%) & $28(22.2)$ & $6(31.6)$ & $22(20.6)$ \\
\hline Treatment fatigue; $n(\%)$ & $27(21.4)$ & $2(10.5)$ & $25(23.4)$ \\
\hline Having problems taking ART; $n(\%)$ & $24(19.0)$ & $3(15.8)$ & $21(19.6)$ \\
\hline Inconvenient clinic operating hours; $n(\%)$ & $21(16.7)$ & $0(0.0)$ & $21(19.6)$ \\
\hline Caregiver financial difficulty; $n(\%)$ & $19(15.1)$ & $3(15.8)$ & $16(15.0)$ \\
\hline Not enough time for visits; $n(\%)$ & $18(14.3)$ & $2(10.5)$ & $16(15.0)$ \\
\hline No sexual health services; $n(\%)$ & $14(11.1)$ & $1(5.3)$ & $13(12.1)$ \\
\hline Caregiver is unsupportive; $n(\%)$ & $13(10.3)$ & $3(15.8)$ & $10(9.3)$ \\
\hline Lack of peer support/counselling; $n(\%)$ & $13(10.3)$ & $5(26.3)$ & $8(7.5)$ \\
\hline Caregiver changes frequently; $n(\%)$ & $8(6.3)$ & $1(5.3)$ & $7(6.5)$ \\
\hline Caregiver is ill and requires care; $n(\%)$ & $8(6.3)$ & $2(10.5)$ & $6(5.6)$ \\
\hline Distrust health care workers; $n(\%)$ & $7(5.6)$ & $1(5.3)$ & $6(5.6)$ \\
\hline Unfriendly health care workers; $n(\%)$ & $4(3.2)$ & $3(15.8)$ & $1(0.9)$ \\
\hline Disinterested in care; $n(\%)$ & $1(0.8)$ & $0(0.0)$ & $1(0.9)$ \\
\hline
\end{tabular}

(Table 3). To determine if perceived barriers to care differed by age group, we stratified the cohort into two categories: younger adolescents aged 12-17 (Supplementary Table A in Supplementary Material available online at http://dx.doi.org/ 10.1155/2016/4161738) and older adolescents aged 18-20 (Supplementary Table B). In addition, having their mother as their primary caregiver appeared to decrease the likelihood of missing a clinic visit after study enrolment (27\% versus $42 \%$ for $12-17$ years old and $39 \%$ versus $55 \%$ for $18-20$ years old). Also greater proportions of adolescents who felt that unfriendly health care workers, having problems in taking the medication, and treatment fatigue were barriers to care missed a subsequent visit compared to those who did not agree with those statements.

Lack of services at health care facilities was also noted by both age groups but the service of importance differed; younger adolescents were more impacted by lack of peer support and counseling (57\% versus $31 \%$ missed visit), while older adolescents agreeing that lack of sexual health services could pose a barrier to care were more likely to miss a subsequent visit (67\% versus $47 \%$ ). Certain potential barriers only appeared to impact adherence to visits in one group and not the other. Among younger adolescents, visits to the clinic being noticed by friends or individuals in the school community were associated with a higher proportion of subsequent missed visits compared to adolescents not perceiving that as a barrier to care ( $46 \%$ versus $27 \%$ ), as was agreeing that difficulty finding time to attend visits and expensive transport fees. For older adolescents, however, agreeing with the statement that having an ill caregiver who requires care $(67 \%$ versus $47 \%$ ) or a caregiver with financial difficulty (71\% versus $43 \%$ ) was barriers to care was more common among those who subsequently missed a clinic visit compared to those who did not agree with those factors as potential barriers.

\section{Discussion}

As pediatric ART has scaled up, HIV care services, mostly delivered in secondary-level health care facilities, are becoming heavily overburdened, while increasing numbers of children are transitioning to adolescence [5]. This is further complicated by the need for skilled health care workers able to manage drug toxicities and psychosocial issues as well as the higher risk of drug resistance which may result from frequent changing of fixed-dose combinations and/or drug stock-outs and lower rates of adherence to medication [32-34].

Few studies have assessed treatment outcomes in older children and/or adolescents in this region, and those that have report disproportionately poor ART outcomes in this group compared to adults. A study investigating treatment outcomes in adolescents (9-19 years) accessing care from a community-based ART program within a periurban township in Cape Town, South Africa, showed that adolescents had significantly lower rates of virologic suppression $(<400$ copies/mL) $(27.3 \%)$ compared to young adults $(63.1 \%)$ [26]. High rates of mortality and loss to follow-up (LTFU) have also been reported among adolescents compared to adults accessing care from primary health care centers [22, $24,26,35]$. A recent study investigating outcomes in multiple HIV cohorts from Gauteng and Mpumalanga in South Africa 
TABLE 3: Factors associated with having at least one late or missed visit $(n=124)$.

\begin{tabular}{|c|c|c|c|}
\hline Characteristic & $N(\%)$ with missed visit & Crude risk ratio $(95 \% \mathrm{CI})$ & Adjusted risk ratio* $(95 \% \mathrm{CI})$ \\
\hline \multicolumn{4}{|c|}{ Baseline and demographic factors } \\
\hline \multicolumn{4}{|c|}{ Gender } \\
\hline Female & $27(39.1)$ & 1.00 & 1.00 \\
\hline Male & $20(36.4)$ & $0.93(0.59-1.47)$ & $1.27(0.76-2.12)$ \\
\hline \multicolumn{4}{|l|}{ Age group } \\
\hline 12-14 years & $15(28.3)$ & 1.00 & 1.00 \\
\hline $15-17$ years & $14(41.2)$ & $1.45(0.81-2.62)$ & $1.80(0.94-3.43)$ \\
\hline $18-20$ years & $18(48.6)$ & $1.72(1.00-2.95)$ & $2.62(0.89-7.67)$ \\
\hline \multicolumn{4}{|c|}{ Highest school level } \\
\hline Secondary & $21(44.7)$ & 1.00 & 1.00 \\
\hline Primary & $22(32.8)$ & $0.73(0.46-1.17)$ & $1.37(0.69-2.72)$ \\
\hline \multicolumn{4}{|l|}{ Dwelling type } \\
\hline Formal & $36(38.3)$ & 1.00 & 1.00 \\
\hline Care facility & $5(38.5)$ & $1.00(0.48-2.09)$ & $0.82(0.40-1.70)$ \\
\hline Informal & $6(35.3)$ & $0.92(0.46-1.84)$ & $1.41(0.65-3.09)$ \\
\hline \multicolumn{4}{|c|}{ Mother as caregiver } \\
\hline No & $27(45.8)$ & 1.00 & 1.00 \\
\hline Yes & $20(30.8)$ & $0.67(0.43-1.06)$ & $0.66(0.43-1.03)$ \\
\hline \multicolumn{4}{|c|}{ Caregiver employed } \\
\hline No & $10(30.3)$ & 1.00 & 1.00 \\
\hline Yes & $32(40.5)$ & $1.34(0.75-2.39)$ & $1.40(0.79-2.48)$ \\
\hline \multicolumn{4}{|l|}{ Time on ART } \\
\hline$<6$ years & $24(40.0)$ & 1.00 & 1.00 \\
\hline$>6$ years & $23(35.9)$ & $0.90(0.57-1.41)$ & $0.98(0.42-2.29)$ \\
\hline \multicolumn{4}{|c|}{ Caregiver-related factors } \\
\hline \multicolumn{4}{|c|}{ Caregiver changes frequently } \\
\hline No & $45(38.8)$ & 1.00 & 1.00 \\
\hline Yes & $2(25.0)$ & $0.64(0.19-2.19)$ & $0.57(0.17-1.88)$ \\
\hline \multicolumn{4}{|c|}{ Caregiver is elderly } \\
\hline No & $30(35.7)$ & 1.00 & 1.00 \\
\hline Yes & $17(42.5)$ & $1.19(0.75-1.89)$ & $1.00(0.63-1.60)$ \\
\hline \multicolumn{4}{|c|}{ Caregiver is ill, requires care } \\
\hline No & $44(37.9)$ & 1.00 & 1.00 \\
\hline Yes & $3(37.5)$ & $0.99(0.39-2.49)$ & $0.94(0.37-2.38)$ \\
\hline \multicolumn{4}{|c|}{ Caregiver is unsupportive } \\
\hline No & $42(37.8)$ & 1.00 & 1.00 \\
\hline Yes & $5(38.5)$ & $1.02(0.49-2.10)$ & $0.82(0.41-1.66)$ \\
\hline \multicolumn{4}{|c|}{ Caregiver financial difficulty } \\
\hline No & $38(36.2)$ & 1.00 & 1.00 \\
\hline Yes & $9(47.4)$ & $1.31(0.76-2.24)$ & $1.29(0.79-2.09)$ \\
\hline \multicolumn{4}{|c|}{ Travel-related factors } \\
\hline \multicolumn{4}{|c|}{ Long distance to clinic } \\
\hline No & $19(40.4)$ & 1.00 & 1.00 \\
\hline Yes & $28(36.4)$ & $0.90(0.57-1.42)$ & $1.00(0.61-1.64)$ \\
\hline \multicolumn{4}{|c|}{ Transport fee is expensive } \\
\hline No & $30(35.7)$ & 1.00 & 1.00 \\
\hline Yes & $17(42.5)$ & $1.19(0.75-1.89)$ & $1.30(0.79-2.13)$ \\
\hline \multicolumn{4}{|c|}{ Not enough time for visits } \\
\hline No & $38(35.5)$ & 1.00 & 1.00 \\
\hline Yes & $9(52.9)$ & $1.49(0.89-2.50)$ & $1.27(0.78-2.09)$ \\
\hline
\end{tabular}


TABLE 3: Continued.

\begin{tabular}{|c|c|c|c|}
\hline Characteristic & $N(\%)$ with missed visit & Crude risk ratio $(95 \% \mathrm{CI})$ & Adjusted risk ratio* $(95 \% \mathrm{CI})$ \\
\hline \multicolumn{4}{|c|}{ Psychosocial factors } \\
\hline \multicolumn{4}{|c|}{ Visits noticed by community } \\
\hline No & $38(39.2)$ & 1.00 & 1.00 \\
\hline Yes & $9(33.3)$ & $0.85(0.47-1.53)$ & $0.75(0.41-1.39)$ \\
\hline \multicolumn{4}{|c|}{ Visits noticed by school/peer } \\
\hline No & $29(34.5)$ & 1.00 & 1.00 \\
\hline Yes & $18(45.0)$ & $1.30(0.83-2.05)$ & $1.21(0.76-1.93)$ \\
\hline \multicolumn{4}{|c|}{ Distrust health care workers } \\
\hline No & $45(38.5)$ & 1.00 & 1.00 \\
\hline Yes & $2(28.6)$ & $0.74(0.23-2.45)$ & $0.84(0.25-2.76)$ \\
\hline \multicolumn{4}{|c|}{ Disclose status to family } \\
\hline No & $3(50.0)$ & 1.00 & 1.00 \\
\hline Yes & $44(37.9)$ & $0.76(0.33-1.75)$ & $0.62(0.26-1.50)$ \\
\hline \multicolumn{4}{|c|}{ Disclose status to school/peer } \\
\hline No & $34(41.0)$ & 1.00 & 1.00 \\
\hline Yes & $13(32.5)$ & $0.79(0.47-1.33)$ & $0.66(0.40-1.09)$ \\
\hline \multicolumn{4}{|c|}{ Health care facility factors } \\
\hline \multicolumn{4}{|c|}{ Long waiting queues at clinic } \\
\hline No & $38(43.7)$ & 1.00 & 1.00 \\
\hline Yes & $9(24.3)$ & $0.56(0.30-1.03)$ & $0.54(0.30-0.99)$ \\
\hline \multicolumn{4}{|c|}{ Inconvenient clinic hours } \\
\hline No & $38(36.9)$ & 1.00 & 1.00 \\
\hline Yes & $9(42.9)$ & $1.16(0.67-2.02)$ & $1.20(0.70-2.05)$ \\
\hline \multicolumn{4}{|c|}{ Unfriendly health care workers } \\
\hline No & $44(36.7)$ & 1.00 & 1.00 \\
\hline Yes & $3(75.0)$ & $2.05(1.11-3.77)$ & $1.61(0.81-3.17)$ \\
\hline \multicolumn{4}{|c|}{ No sexual health services } \\
\hline No & $41(37.3)$ & 1.00 & 1.00 \\
\hline Yes & $6(42.9)$ & $1.15(0.60-2.21)$ & $1.26(0.69-2.30)$ \\
\hline \multicolumn{4}{|c|}{ Lack of peer support } \\
\hline No & $41(36.9)$ & 1.00 & 1.00 \\
\hline Yes & $6(46.2)$ & $1.25(0.66-2.36)$ & $0.93(0.48-1.82)$ \\
\hline \multicolumn{4}{|c|}{ Treatment-related factors } \\
\hline \multicolumn{4}{|c|}{ Having problems taking ART } \\
\hline No & $33(33.0)$ & 1.00 & 1.00 \\
\hline Yes & $14(58.3)$ & $1.77(1.14-2.74)$ & $1.45(0.91-2.32)$ \\
\hline \multicolumn{4}{|c|}{ Treatment fatigue } \\
\hline No & $32(32.7)$ & 1.00 & 1.00 \\
\hline Yes & $15(57.7)$ & $1.77(1.14-2.73)$ & $1.47(0.94-2.31)$ \\
\hline
\end{tabular}

${ }^{*}$ Risk ratios individually adjusted for age, gender, mother as caregiver, and time on ART.

found that attrition in ART care occurred soon after the commencement of ART; the median time to death or loss to care was 4.7 months (IQR: $1.5-13.2$ ) and 10.9 months (IQR: 5.0-22.7), respectively. Adolescents were more likely to be LTFU after ART initiation (hazard ratio $(\mathrm{HR})=1.38$; 95\% CI: 1.07-1.78) compared to adults, though little age difference in mortality was found [25]. Despite this clear indication of increased risk for poor treatment outcomes among adolescents, the potential causes of poor adherence to treatment are yet to be clearly identified.
We interviewed a group of 126 adolescents and aimed to uncover what factors adolescents perceived to be barriers to accessing and remaining in care. Further, we estimated associations between agreeing that a factor was a barrier to care and subsequently missing a scheduled HIV clinic visit and whether these differed between older and younger adolescents. We found that the most frequently reported perceived barriers to care were related to logistics of the actual clinic visit: time and cost involved in getting to the visit as well as duration of time spent waiting in queues. Treatment 
TABLE 4: Factors associated with prevalent virologic failure among adolescents with a recorded VL measurement $(n=103)$.

\begin{tabular}{|c|c|c|c|}
\hline Variable & $n$ (\%) with prevalent VL failure & Crude risk ratio $(95 \% \mathrm{CI})$ & Adjusted* risk ratio (95\% CI) \\
\hline \multicolumn{4}{|c|}{ Baseline and demographic factors } \\
\hline \multicolumn{4}{|c|}{ Gender } \\
\hline Female & $16(27.1 \%)$ & 1.00 & 1.00 \\
\hline Male & $16(36.4 \%)$ & $1.54(0.66-3.56)$ & $1.44(0.76-2.73)$ \\
\hline \multicolumn{4}{|c|}{ Age group (years) } \\
\hline $12-14$ & $11(25.6 \%)$ & 1.00 & 1.00 \\
\hline $15-17$ & $11(37.9 \%)$ & $1.78(0.64-4.91)$ & $2.67(0.76-9.34)$ \\
\hline $18-20$ & $10(32.3 \%)$ & $1.39(0.50-3.83)$ & $2.77(0.51-14.96)$ \\
\hline \multicolumn{4}{|c|}{ Highest school level } \\
\hline Secondary & $14(35.0 \%)$ & 1.00 & 1.00 \\
\hline Primary & $14(25.9 \%)$ & $0.65(0.27-1.58)$ & $1.17(0.28-4.79)$ \\
\hline \multicolumn{4}{|l|}{ Dwelling type } \\
\hline Formal & $26(32.9 \%)$ & 1.00 & - \\
\hline Care facility & $1(9.1 \%)$ & $0.20(0.02-1.68)$ & - \\
\hline Informal & $5(38.5 \%)$ & $1.27(0.38-4.28)$ & - \\
\hline \multicolumn{4}{|c|}{ Mother caregiver } \\
\hline No & $15(31.3 \%)$ & 1.00 & - \\
\hline Yes & $17(30.9 \%)$ & $0.98(0.43-2.27)$ & - \\
\hline \multicolumn{4}{|c|}{ Caregiver employed } \\
\hline No & $10(35.7 \%)$ & 1.00 & - \\
\hline Yes & $21(32.3 \%)$ & $0.86(0.34-2.18)$ & - \\
\hline \multicolumn{4}{|c|}{ Caregiver-related factors } \\
\hline \multicolumn{4}{|c|}{ Caregiver changes frequently } \\
\hline No & $32(32.7 \%)$ & 1.00 & - \\
\hline Yes & $0(0.0 \%)$ & - & - \\
\hline \multicolumn{4}{|c|}{ Caregiver is elderly } \\
\hline No & $22(30.6 \%)$ & 1.00 & 1.00 \\
\hline Yes & $10(32.3 \%)$ & $1.08(0.44-2.67)$ & $1.31(0.69-2.48)$ \\
\hline \multicolumn{4}{|c|}{ Caregiver is ill, requires care } \\
\hline No & $29(29.9 \%)$ & 1.00 & 1.00 \\
\hline Yes & $3(50.0 \%)$ & $2.34(0.45-12.31)$ & $0.65(0.09-4.55)$ \\
\hline \multicolumn{4}{|c|}{ Caregiver is unsupportive } \\
\hline No & $28(30.8 \%)$ & 1.00 & - \\
\hline Yes & $4(33.3 \%)$ & $1.13(0.31-4.05)$ & - \\
\hline \multicolumn{4}{|c|}{ Caregiver financial difficulty } \\
\hline No & $26(30.2 \%)$ & 1.00 & 1.00 \\
\hline Yes & $6(35.3 \%)$ & $1.26(0.42-3.77)$ & $2.51(0.51-12.38)$ \\
\hline \multicolumn{4}{|c|}{ Travel-related factors } \\
\hline \multicolumn{4}{|c|}{ Long distance to clinic } \\
\hline No & $11(28.2 \%)$ & 1.00 & 1.00 \\
\hline Yes & $21(32.8 \%)$ & $1.24(0.52-2.97)$ & $0.43(0.18-1.04)$ \\
\hline \multicolumn{4}{|c|}{ Transport fee is expensive } \\
\hline No & $20(27.8 \%)$ & 1.00 & 1.00 \\
\hline Yes & $12(38.7 \%)$ & $1.64(0.68-3.99)$ & $1.79(0.76-4.19)$ \\
\hline \multicolumn{4}{|c|}{ Not enough time for visits } \\
\hline No & $28(31.1 \%)$ & 1.00 & - \\
\hline Yes & $4(30.8 \%)$ & $0.98(0.28-3.47)$ & - \\
\hline \multicolumn{4}{|c|}{ Psychosocial factors } \\
\hline \multicolumn{4}{|c|}{ Visits noticed by community } \\
\hline No & $32.9 \%(26)$ & 1.00 & 1.00 \\
\hline Yes & $25.0 \%(6)$ & $0.68(0.24-1.92)$ & $0.37(0.15-0.93)$ \\
\hline
\end{tabular}


TABLE 4: Continued.

\begin{tabular}{|c|c|c|c|}
\hline Variable & $n$ (\%) with prevalent VL failure & Crude risk ratio (95\% CI) & Adjusted ${ }^{*}$ risk ratio $(95 \% \mathrm{CI})$ \\
\hline \multicolumn{4}{|c|}{ Visits noticed by school/peer } \\
\hline No & $31.4 \%(22)$ & 1.00 & 1.00 \\
\hline Yes & $30.3 \%(10)$ & $0.95(0.39-2.33)$ & $1.02(0.44-2.37)$ \\
\hline \multicolumn{4}{|c|}{ Distrust health care workers } \\
\hline No & $30.3 \%(30)$ & 1.00 & 1.00 \\
\hline Yes & $50.0 \%(2)$ & $2.30(0.31-17.10)$ & $6.15(1.14-33.24)$ \\
\hline \multicolumn{4}{|c|}{ Disclose status to family } \\
\hline No & $33.3 \%(1)$ & 1.00 & 1.00 \\
\hline Yes & $31.3 \%(31)$ & $0.91(0.08-10.44)$ & $1.34(0.39-4.63)$ \\
\hline \multicolumn{4}{|c|}{ Disclose status to school/peer } \\
\hline No & $34.3 \%(24)$ & 1.00 & 1.00 \\
\hline Yes & $25.0 \%(8)$ & $0.64(0.25-1.64)$ & $0.51(0.20-1.26)$ \\
\hline \multicolumn{4}{|c|}{ Health care facility factors } \\
\hline \multicolumn{4}{|c|}{ Long waiting queues at clinic } \\
\hline No & $27(35.5 \%)$ & 1.00 & 1.00 \\
\hline Yes & $5(18.5 \%)$ & $0.41(0.14-1.21)$ & $0.94(0.28-3.12)$ \\
\hline \multicolumn{4}{|c|}{ Inconvenient clinic operating hours } \\
\hline No & $28(32.6 \%)$ & 1.00 & 1.00 \\
\hline Yes & $4(23.5 \%)$ & $0.64(0.19-2.13)$ & $0.49(0.16-1.52)$ \\
\hline \multicolumn{4}{|c|}{ Unfriendly health care workers } \\
\hline No & $32(31.4 \%)$ & 1.00 & - \\
\hline Yes & $0(0.0 \%)$ & - & - \\
\hline \multicolumn{4}{|c|}{ No sexual health services } \\
\hline No & $28(31.1 \%)$ & 1.00 & 1.00 \\
\hline Yes & $4(30.8 \%)$ & $0.98(0.28-3.47)$ & $0.70(0.19-2.50)$ \\
\hline \multicolumn{4}{|c|}{ Lack of peer support } \\
\hline No & $31(33.0 \%)$ & 1.00 & - \\
\hline Yes & $1(11.1 \%)$ & $0.25(0.03-2.12)$ & - \\
\hline \multicolumn{4}{|c|}{ Treatment-related factors } \\
\hline \multicolumn{4}{|c|}{ Having problems taking ART } \\
\hline No & $26(31.3 \%)$ & 1.00 & 1.00 \\
\hline Yes & $6(30.0 \%)$ & $0.94(0.32-2.72)$ & $0.36(0.16-0.82)$ \\
\hline \multicolumn{4}{|c|}{ Treatment fatigue } \\
\hline No & $23(28.4 \%)$ & 1.00 & 1.00 \\
\hline Yes & $9(40.9 \%)$ & $1.75(0.66-4.64)$ & $1.71(0.78-3.75)$ \\
\hline
\end{tabular}

${ }^{*}$ Risk ratios individually adjusted for age, gender, mother as caregiver, and time on ART.

programs requiring patients to return to the clinic more frequently or travel longer distances to receive necessary drugs increase the economic burden of transport costs and the possibility of lost wages for patients, both of which are well known barriers of adherence to HIV care and treatment $[13,14]$. Though our sample was restricted to an urban setting, previous research has also shown that the type of community (urban versus rural) can be a factor of adherence to HIV care and treatment due to differences in community characteristics such as density of population, distance to and availability of clinics and hospitals, and infrastructure within clinics [36, 37]. Reassuringly, very few considered not being interested in treatment, unfriendly health care workers, or distrust of health care workers as important potential barriers to care, though it must be considered that adolescent experiencing these barriers personally may not be attending visits at all.

We also found that older adolescents appear to be a key subgroup of this already vulnerable population; older adolescents were at increased risk for missing a clinic visit and were also more likely to have experienced prevalent virologic failure at the time of study enrolment compared to younger age categories. This has been demonstrated elsewhere $[24,25$, 38,39 ] and understanding drivers of poor adherence in this group is critical. One possibility is simply that younger age groups represent perinatally infected children who represent a group who have been on treatment for longer period of time and are more likely to be adherent to treatment. However, the reality for many adolescents accessing care in this setting is 
that attending a clinic visit may take up a full day requiring time off school as well as time away from other household and family responsibilities. The impact of this time pressure does appear to differ between older and younger adolescents. We note that older adolescents more frequently agreed that having an elderly caregiver presented a potential barrier to care $(47 \%)$ compared to younger adolescents (33\%). This may reflect social circumstances where, as adolescent ages, increased responsibilities for caring for elderly members of the family are placed on them, in which, along with increasing demands during more senior years at school or entry into the workforce, time away from school/work and home responsibilities may represent a substantial obstacle in this group. While having problems in taking the medication and treatment fatigue were identified as barriers in both younger and older adolescents who missed a visit, the proportion of older adolescents that identified unfriendly health care workers as a barrier was far greater (RR: 2.19; 95\% CI: 1.52-3.14, Table 3 ). Though the numbers represented are too small to make strong inferences, differences in provider relationships between adult-based and pediatric-based facilities could be important and modifiable factors affecting adherence to care.

Having problems taking ART (most frequently reported as forgetting to take the medication) was identified as one of the treatment-related barriers to care by both age groups of adolescents and was associated with an increased risk of poor adherence to visits. Several interventions have been tested for feasibility and efficacy in terms of reminders to take tablets and may present an opportunity to improve a relatively easily modifiable risk factor for poor adherence among adolescents. These include mobile phone text message reminders and electronic medication monitoring devices [40, 41]. Though adolescents frequently agreed that treatment fatigue was a potential barrier to accessing and remaining in care and those who agreed with this statement were more likely to subsequently miss a visit (58\% versus 33\%), there was little difference in the likelihood of missing visits by time on ART ( $40 \%$ and $36 \%$ for those on ART $<6$ years and $>6$ years, resp.). It is possible that attrition due to treatment fatigue occurs earlier on and the group that would be susceptible to dropping out of care due to treatment fatigue is not accurately represented in this study.

Disclosure of HIV status is an important issue in adolescent populations: not only disclosure of status to the vertically infected child but also disclosure by the adolescents themselves to family, friends, and members of their school and community. Among the group of adolescents interviewed, $>95 \%$ agreed that disclosing status to family would facilitate access to care, but only a third agreed that disclosing to school and friends would be of assistance. In fact, one of the most frequently perceived barriers to care was the fact that the frequency of HIV clinic visits would be noticed by friends or members of the school community and among the group aged 12-17; perceiving this factor as a barrier to care was associated with a $70 \%$ increased risk of subsequently missing a clinic visit, underlining the social challenges and stigma that school-going adolescents face while in ART care programs.

Our findings must be considered in light of some limitations. First, we acknowledge that, by definition, all the participants interviewed were still accessing care at the time of the study and the factors identified by this group as important determinants of accessing and staying in care may differ from those that would potentially be identified by adolescents who have already dropped out of care. Second, the small numbers enrolled limited the statistical power to detect small differences and precision of our estimates. However, the analysis presented here aimed to explore possible associations between the factors investigated and adherence to ART treatment rather than establishing causal associations and should be interpreted as such. Third, we were unable to enroll some participants $<18$ years of age ( $9 \%$ of the total screened participants) due to the fact that a legal guardian was not able to be present to sign consent. If those who were not enrolled differed systematically from those $<18$ years that consented, this could result in selection bias. In addition, bias may arise if the need for caregiver consent to participate in the study influenced the responses provided by the adolescents interviewed. Though we cannot rule this possibility out completely, several steps were taken during the consenting and interviewing process to prevent this, including an explanation of the procedures undertaken to ensure confidentiality and conducting the interview in a private space. Finally, we did not measure depression or stigma directly as potential barriers to care and these may contribute significantly to the perception of other factors.

\section{Conclusions}

Despite these limitations, our study results are important. First, we demonstrate the increased risk of poor adherence to care for older adolescents, highlighting this group as a key population for intervention if 90-90-90 targets are to be met. In addition, we report on several modifiable factors that may be barriers to accessing and remaining in care among adolescents and show that these differ in importance and impact between older and younger adolescents. Several different interventions to improve retention in care and adherence to treatment have been proposed including financial incentives and behavioral and facility level interventions such as peer social support and adherence clubs [35, 42-46]. Evaluation of the effectiveness and potential impact of different intervention approaches is needed within the context of each of the progressive stages of adolescence. Adherence to ART is complex, and a multifaceted approach acknowledging changing barriers to accessing and remaining in care is required when designing and implementing interventions for adolescent populations.

\section{Disclosure}

The contents are the responsibility of the authors and do not necessarily reflect the views of USAID, the Fogarty International Centre, or the United States government.

\section{Competing Interests}

The authors declare that there are no competing interests regarding the publication of this paper. 


\section{Acknowledgments}

The study team would like to acknowledge and thank the patients and clinical staff at the study sites who made this work possible. This study was also made possible by the generous support of the American people through the United States Agency for International Development (INROADS USAID-674-A-12-00029) and Fogarty International Centre (ICORTA Grant 3U2RTW007370-05S1).

\section{References}

[1] UNAIDS, "90-90-90 An ambitious treatment target to help end the AIDS epidemic," 2014, http://www.unaids.org/sites/default/ files/media_asset/90-90-90_en_0.pdf.

[2] National Department of Health South Africa, HIV \& AIDS and STI National Strategic Plan 2007-2011, 2007.

[3] National Department of Health South Africa, National Strategic Plan on HIV, STIs and TB: 2012-2016, National Department of Health South Africa, Pretoria, South Africa, 2012.

[4] UNICEF, "UNICEF Data: Adolescents and Young People Current Status and Progress," 2016, http://data.unicef.org/hiv-aids/ adolescents-young-people.html.

[5] R. A. Ferrand, E. L. Corbett, R. Wood et al., "AIDS among older children and adolescents in Southern Africa: projecting the time course and magnitude of the epidemic," AIDS, vol. 23, no. 15, pp. 2039-2046, 2009.

[6] A. M. Doyle, S. N. Mavedzenge, M. L. Plummer, and D. A. Ross, "The sexual behaviour of adolescents in sub-Saharan Africa: patterns and trends from national surveys," Tropical Medicine and International Health, vol. 17, no. 7, pp. 796-807, 2012.

[7] PEPFAR, "DREAMS: Working together for an AIDS-free future for girls \& women," 2015.

[8] E. Robson, N. Ansell, U. S. Huber, W. T. S. Gould, and L. van Blerk, "Young caregivers in the context of the HIV/AIDS pandemic in sub-Saharan Africa," Population, Space and Place, vol. 12, no. 2, pp. 93-111, 2006.

[9] G. Andrews, D. Skinner, and K. Zuma, "Epidemiology of health and vulnerability among children orphaned and made vulnerable by HIV/AIDS in sub-Saharan Africa," AIDS Care, vol. 18, no. 3, pp. 269-276, 2006.

[10] G. Foster and J. Williamson, "A review of current literature on the impact of HIV/AIDS on children in sub-Saharan Africa," AIDS, vol. 14, supplement 3, pp. S275-S284, 2000.

[11] S. M. Sawyer, R. A. Afifi, L. H. Bearinger et al., "Adolescence: a foundation for future health," The Lancet, vol. 379, no. 9826, pp. 1630-1640, 2012.

[12] National Department of Health, The South African Antiretroviral Treatment Guidelines, South Africa, 2010.

[13] E. H. Geng, D. R. Bangsberg, N. Musinguzi et al., "Understanding reasons for and outcomes of patients lost to follow-up in antiretroviral therapy programs in Africa through a samplingbased approach," Journal of Acquired Immune Deficiency Syndromes, vol. 53, no. 3, pp. 405-411, 2010.

[14] B. Amuron, G. Namara, J. Birungi et al., "Mortality and loss-tofollow-up during the pre-treatment period in an antiretroviral therapy programme under normal health service conditions in Uganda," BMC Public Health, vol. 9, article 290, 2009.
[15] L. F. Langhaug, S. J. Pascoe, W. Mavhu et al., "High prevalence of affective disorders among adolescents living in Rural Zimbabwe," Journal of Community Health, vol. 35, no. 4, pp. 355364, 2010.

[16] P. M. Godia, J. M. Olenja, J. A. Lavussa, D. Quinney, J. J. Hofman, and N. van den Broek, "Sexual reproductive health service provision to young people in Kenya; health service providers' experiences," BMC Health Services Research, vol. 13, no. 1, article 476, 2013.

[17] H. B. Jaspan, R. Li, L. Johnson, and L.-G. Bekker, "The emerging need for adolescent-focused HIV care in South Africa," Southern African Journal of HIV Medicine, no. 36, pp. 9-11, 2009.

[18] UNAIDS, Global AIDS Update, UNAIDS, Geneva, Switzerland, 2016.

[19] National Department of Health South Africa, HIV \& AIDS 2014/15 Data fact sheet, June 2015.

[20] World Health Organisation, Global HIV/AIDS Response: Epidemic update and health sector progress towards Universal Access, Progress Report, 2011, http://www.who.int/hiv/pub/ progress_report2011/hiv_full_report_2011.pdf.

[21] USAID, HIV and AIDS Estimates, USAID, Washington, DC, USA, 2014.

[22] H. Bygrave, J. Mtangirwa, K. Ncube, N. Ford, K. Kranzer, and D. Munyaradzi, "Antiretroviral therapy outcomes among adolescents and youth in rural Zimbabwe," PLoS ONE, vol. 7, no. 12, Article ID e52856, 2012.

[23] P. Bock, A. Boulle, C. White, M. Osler, and B. Eley, "Provision of antiretroviral therapy to children within the public sector of South Africa," Transactions of the Royal Society of Tropical Medicine and Hygiene, vol. 102, no. 9, pp. 905-911, 2008.

[24] J. B. Nachega, M. Hislop, H. Nguyen et al., "Antiretroviral therapy adherence, virologic and immunologic outcomes in adolescents compared with adults in Southern Africa," Journal of Acquired Immune Deficiency Syndromes, vol. 51, no. 1, pp. 6571, 2009.

[25] D. Evans, C. Menezes, K. Mahomed et al., “Treatment outcomes of HIV-infected adolescents attending public-sector HIV clinics across Gauteng and Mpumalanga, South Africa," AIDS Research and Human Retroviruses, vol. 29, no. 6, pp. 892-900, 2013.

[26] M. D. Nglazi, K. Kranzer, P. Holele et al., “Treatment outcomes in HIV-infected adolescents attending a community-based antiretroviral therapy clinic in South Africa," BMC Infectious Diseases, vol. 12, no. 1, article 21, 2012.

[27] M. R. Lamb, R. Fayorsey, H. Nuwagaba-Biribonwoha et al., "High attrition before and after ART initiation among youth (15-24 years of age) enrolled in HIV care," AIDS, vol. 28, no. 4, pp. 559-568, 2014.

[28] M. Maskew, J. Bor, W. MacLeod, S. Carmona, G. Sherman, and M. P. Fox, "Youth treatment bulge in South Africa: increasing numbers, inferior outcomes among adolescents on ART," in Proceedings of the 21st International AIDS Conference, Durban, South Africa, July 2016.

[29] J. Watermeyer, “This clinic is number one': a qualitative study of factors that contribute toward 'successful' care at a South African pediatric HIV/AIDS clinic," Evaluation and the Health Professions, vol. 35, no. 3, pp. 360-379, 2012.

[30] K.-G. Technau, E. Lazarus, L. Kuhn et al., "Poor early virologic performance and durability of abacavir-based first-line regimens for HIV-infected children," Pediatric Infectious Disease Journal, vol. 32, no. 8, pp. 851-855, 2013. 
[31] M. P. Fox, M. Maskew, A. P. MacPhail et al., "Cohort profile: the Themba Lethu Clinical Cohort, Johannesburg, South Africa," International Journal of Epidemiology, vol. 42, no. 2, pp. 430439, 2013.

[32] R. M. Bland, "Management of HIV-infected children in Africa: progress and challenges," Archives of Disease in Childhood, vol. 96, no. 10, pp. 911-915, 2011.

[33] A. J. De Baets, M. Bulterys, E. J. Abrams, C. Kankassa, and I. E. Pazvakavambwa, "Care and treatment of HIV-infected children in Africa: issues and challenges at the district hospital level," Pediatric Infectious Disease Journal, vol. 26, no. 2, pp. 163-173, 2007.

[34] T. Meyers, H. Moultrie, K. Naidoo, M. Cotton, B. Eley, and G. Sherman, "Challenges to pediatric HIV care and treatment in South Africa," Journal of Infectious Diseases, vol. 196, no. 3, pp. S474-S481, 2007.

[35] A. K. Chan, G. Mateyu, A. Jahn et al., "Outcome assessment of decentralization of antiretroviral therapy provision in a rural district of Malawi using an integrated primary care model," Tropical Medicine and International Health, vol. 15, supplement 1, pp. 90-97, 2010.

[36] M. Lahuerta, J. Lima, H. Nuwagaba-Biribonwoha et al., "Factors associated with late antiretroviral therapy initiation among adults in Mozambique," PloS ONE, vol. 7, no. 5, Article ID e37125, 2012.

[37] B. A. Larson, A. Brennan, L. McNamara et al., "Lost opportunities to complete CD4+ lymphocyte testing among patients who tested positive for HIV in South Africa," Bulletin of the World Health Organization, vol. 88, no. 9, pp. 675-680, 2010.

[38] S. Y. Kahana, J. Rohan, S. Allison, T. W. Frazier, and D. Drotar, "A meta-analysis of adherence to antiretroviral therapy and virologic responses in HIV-infected children, adolescents, and young adults," AIDS and Behavior, vol. 17, no. 1, pp. 41-60, 2013.

[39] L. Fairlie, B. Karalius, K. Patel et al., "CD4 ${ }^{+}$and viral load outcomes of antiretroviral therapy switch strategies after virologic failure of combination antiretroviral therapy in perinatally HIVinfected youth in the United States," AIDS, vol. 29, no. 16, pp. 2109-2119, 2015.

[40] P. Saberi, K. Mayer, E. Vittinghoff, S. Naar-King, and Adolescent Medicine Trials Network for HIV/AIDS Interventions, "Correlation between use of antiretroviral adherence devices by HIV-infected youth and plasma HIV RNA and self-reported adherence," AIDS and Behavior, vol. 19, no. 1, pp. 93-103, 2014.

[41] C. Orrell, K. Cohen, K. Mauff, D. R. Bangsberg, G. Maartens, and R. Wood, "A randomized controlled trial of real-time electronic adherence monitoring with text message dosing reminders in people starting first-line antiretroviral therapy," Journal of Acquired Immune Deficiency Syndromes, vol. 70, no. 5, pp. 495-502, 2015.

[42] J. S. Cervia, "Easing the transition of HIV-infected adolescents to adult care," AIDS Patient Care and STDs, vol. 27, no. 12, pp. 692-696, 2013.

[43] N. Dowshen and L. D'Angelo, "Health care transition for youth living with HIV/AIDS," Pediatrics, vol. 128, no. 4, pp. 762-771, 2011.

[44] C. D. Fair, K. Sullivan, and A. Gatto, "Indicators of transition success for youth living with HIV: perspectives of pediatric and adult infectious disease care providers," AIDS Care, vol. 23, no. 8, pp. 965-970, 2011.

[45] C. A. Teasdale, T. Alwar, D. Chege, R. Fayorsey, M. P. Hawken, and E. J. Abrams, "Impact of youth and adolescent friendly services on retention of 10-24-year-olds in HIV Care and treatment programs in Nyanza, Kenya," Journal of Acquired Immune Deficiency Syndromes, vol. 71, no. 2, pp. e56-e59, 2016.

[46] T. Decroo, O. Koole, D. Remartinez et al., "Four-year retention and risk factors for attrition among members of community ART groups in Tete, Mozambique," Tropical Medicine and International Health, vol. 19, no. 5, pp. 514-521, 2014. 


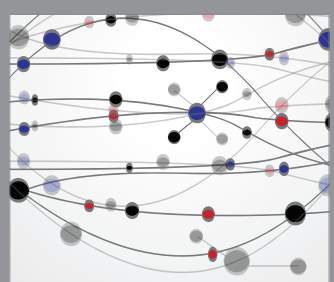

The Scientific World Journal
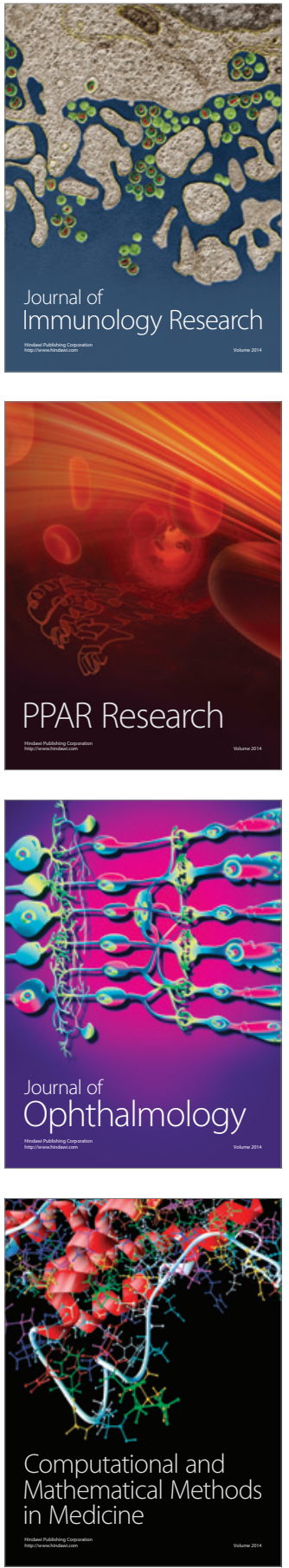

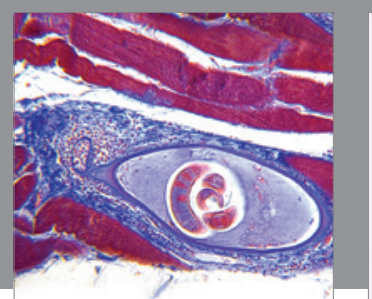

Gastroenterology Research and Practice

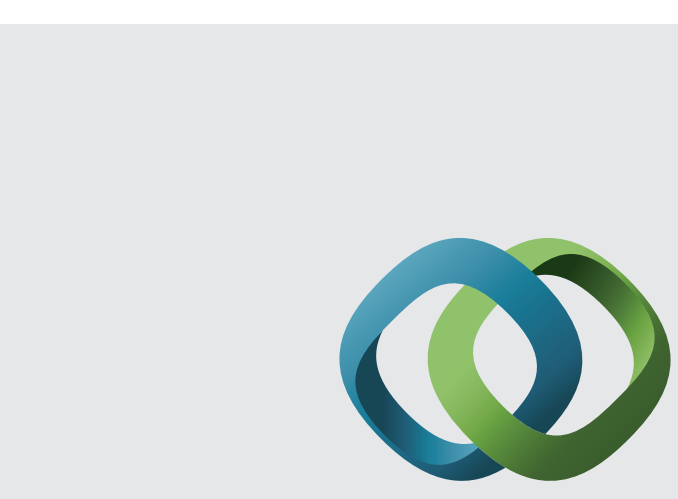

\section{Hindawi}

Submit your manuscripts at

http://www.hindawi.com
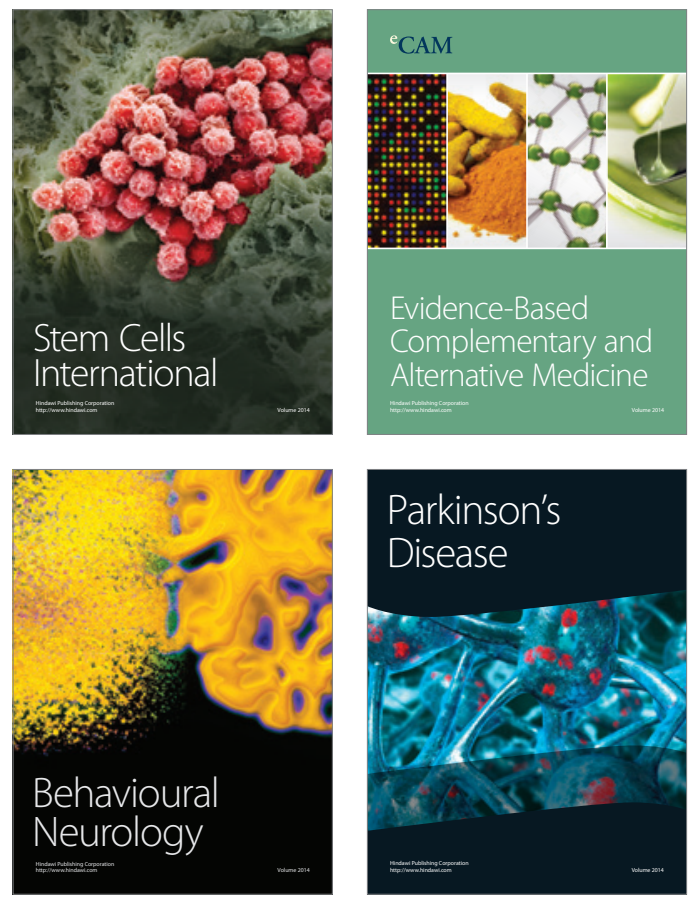
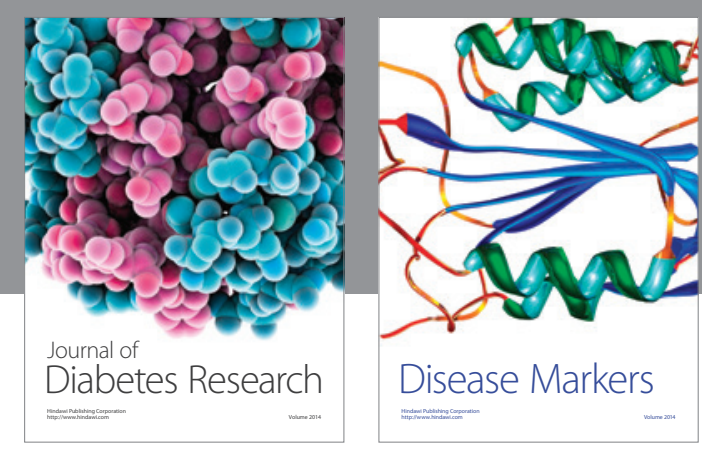

Disease Markers
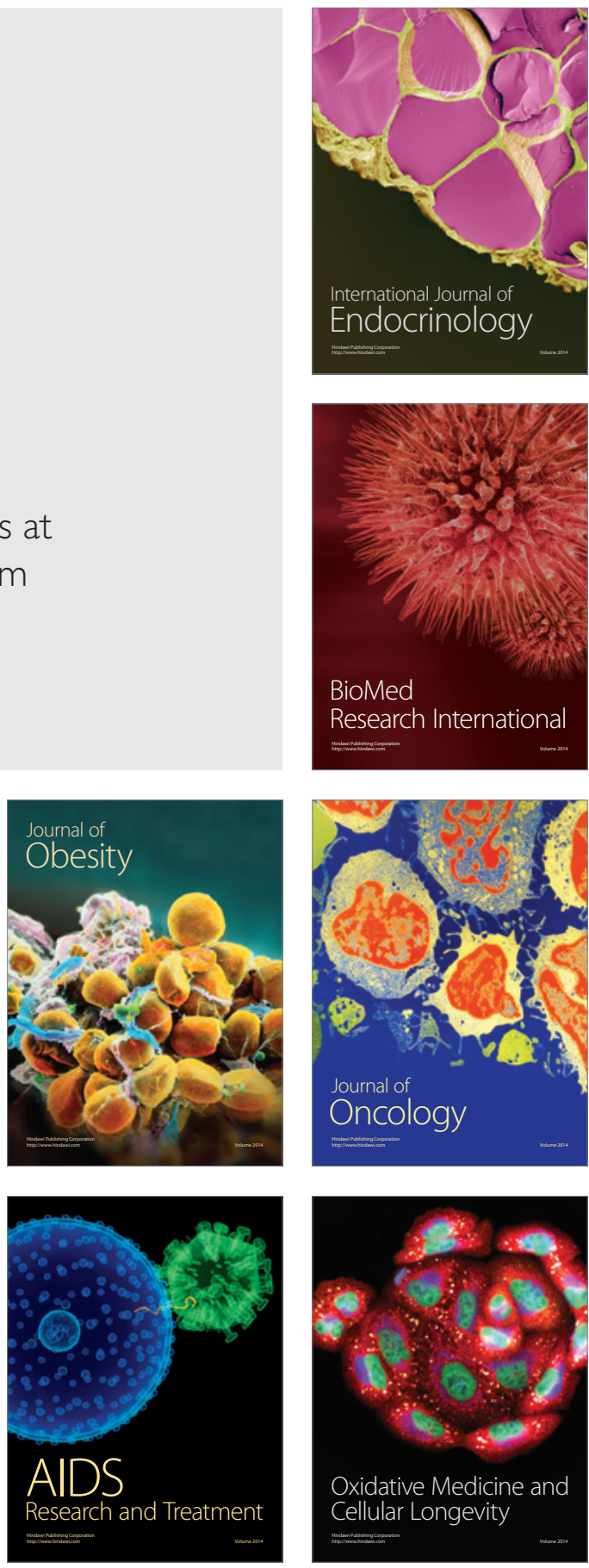